\title{
Modeling of weak-lensing statistics
}

\section{Configuration-space statistics}

\author{
P. Valageas ${ }^{1}$, M. Sato ${ }^{2}$, and T. Nishimichi ${ }^{3}$ \\ 1 Institut de Physique Théorique, CEA Saclay, 91191 Gif-sur-Yvette, France \\ e-mail: patrick.valageas@cea.fr \\ 2 Department of Physics, Nagoya University, 464-8602 Nagoya, Japan \\ ${ }^{3}$ Institute for the Physics and Mathematics of the Universe, University of Tokyo, Kashiwa, 277-8568 Chiba, Japan \\ Received 5 December 2011 / Accepted 30 January 2012
}

\section{ABSTRACT}

\begin{abstract}
Aims. We investigate the performance of an analytic model of the 3D matter distribution, which combines perturbation theory with halo models, for weak-lensing configuration-space statistics.

Methods. We compared our predictions for the weak-lensing convergence two-point and three-point correlation functions with numerical simulations and fitting formulas proposed in previous works. We also considered the second- and third-order moments of the smoothed convergence and of the aperture-mass.

Results. As in our previous study of Fourier-space weak-lensing statistics, we find that our model agrees better with simulations than previously published fitting formulas. Moreover, we recover the dependence on cosmology of these weak-lensing statistics and we can describe multi-scale moments. This approach allows us to obtain the quantitative relationship between these integrated weak-lensing statistics and the various contributions to the underlying 3D density fluctuations, decomposed over perturbative, two-halo, or one-halo terms.
\end{abstract}

Key words. gravitational lensing: weak - large-scale structure of Universe

\section{Introduction}

The standard paradigm known as the $\Lambda$ CDM cosmology includes dark components: dark matter and dark energy (Komatsu et al. 2011). Weak lensing of background galaxies by foreground large-scale structures, the so-called "cosmic shear", has been recognized as a potentially powerful tool for probing the distribution of dark matter as well as the nature of dark energy (Albrecht et al. 2006). Reports of significant detections of cosmic shear have been made by various groups (Bacon et al. 2000; Van Waerbeke et al. 2000; Wittman et al. 2000; Hamana et al. 2003; Jarvis et al. 2006; Semboloni et al. 2006; Fu et al. 2008; Schrabback et al. 2010).

By analyzing the cosmic shear data, one can directly measure the power spectrum of the matter density fluctuations on cosmological scales, which contain a wealth of cosmological information such as neutrino masses and dark energy equationof-state parameters (Jarvis et al. 2006; Semboloni et al. 2006; Ichiki et al. 2009; Schrabback et al. 2010). Consequently, it is a main goal of cosmology to infer and constrain these quantities from observations. To do this, a number of ambitious surveys are planned, such as the Hyper Suprime-Cam Weak Lensing Survey (Miyazaki et al. 2006) ${ }^{1}$, the Dark Energy Survey $(\mathrm{DES})^{2}$, the Large Synoptic Survey Telescope (LSST) ${ }^{3}$,

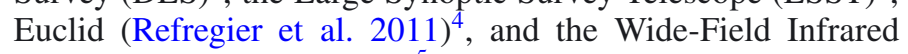
Survey Telescope (WFIRST) ${ }^{5}$.

\footnotetext{
${ }^{1}$ http://www.naoj.org/Projects/HSC/index.html

2 http://www. darkenergysurvey.org/

3 http://www. lsst.org/

${ }^{4}$ http://www. euclid-ec.org/

5 http://wfirst.gsfc.nasa.gov/
}

Most weak-lensing information is contained in small angular scales and therefore weak-lensing statistics are nonlinear and non-Gaussian (Munshi et al. 2008; Takada \& Jain 2009; Sato et al. 2009, 2011b). If we aim to exploit the full information, we have to treat the nonlinear effects to accurately model the weak lensing statistics. Furthermore, one has to use an appropriate likelihood function with given marginal distributions, otherwise the obtained results would be systematically biased (Sato et al. 2010, 2011a).

In a first companion paper (Valageas et al. 2012, hereafter Paper I), we studied the Fourier-space weak-lensing statistics such as the weak-lensing power spectrum and bispectrum, and found that our model proposed by Valageas \& Nishimichi (2011a,b), which combines perturbation theory with halo models, agrees better with ray-tracing simulations than previously published fitting formulas and phenomenological models. In this second paper, we study the real-space weak-lensing statistics, which are more often used for the statistical analysis of actual measurements than Fourier-space statistics, because observations are made in configuration space.

Previous works have already shown that on small scales the halo model provides a good description of the two-, threeand four-point correlations or smoothed moments of the cosmic shear (using some approximations) (Takada \& Jain 2002, 2003; Benabed \& Scoccimarro 2006), whereas a stochastic halo model can recover the probability distribution function of the unsmoothed convergence (Kainulainen \& Marra 2011a,b). Here we include all "one-halo", "two-halo" and "three-halo" terms, as well as one-loop perturbative results, and we compare these with larger-scale simulations. This yields a greater accuracy and allows us to compare these different contributions, from very large to small scales. This should be useful for practical purpose 
because these different terms have different theoretical accuracies and probe different regimes of gravitational clustering, hence it is important to know their relative impact on weaklensing probes as a function of angular scale.

This paper is organized as follows. In Sect. 2 we briefly recall how configuration-space weak-lensing statistics are computed from polyspectra of the 3D matter density field. We describe our numerical simulations and the data analysis in Sect. 3. Then, we present detailed comparisons between the simulation results, previous models, and our theoretical predictions for twopoint functions in Sect. 4 and three-point functions in Sect. 5. We study the relative importance of the different contributions arising from "one-halo", "two-halo", or "three-halo" terms in Sect. 6. Then, we check the robustness of our model when we vary the cosmological parameters in Sect. 7 and we briefly study multi-scale moments in Sect. 8. Finally, we conclude in Sect. 9.

\section{From 3D statistics to weak-lensing statistics}

\subsection{Lensing power spectrum and bispectrum}

Using Born's approximation, the weak-lensing convergence $\kappa(\boldsymbol{\theta})$ can be written as the integral of the density contrast along the line of sight (Bartelmann \& Schneider 2001; Munshi et al. 2008),

$\kappa(\boldsymbol{\theta})=\int_{0}^{\chi_{\mathrm{s}}} \mathrm{d} \chi w\left(\chi, \chi_{\mathrm{s}}\right) \delta(\chi, \mathcal{D} \boldsymbol{\theta})$

where $\chi$ and $\mathcal{D}$ are the radial and angular comoving distances,

$w\left(\chi, \chi_{\mathrm{s}}\right)=\frac{3 \Omega_{\mathrm{m}} H_{0}^{2} \mathcal{D}(\chi) \mathcal{D}\left(\chi_{\mathrm{s}}-\chi\right)}{2 c^{2} \mathcal{D}\left(\chi_{\mathrm{s}}\right)}(1+z)$,

and $z_{\mathrm{s}}$ is the redshift of the source (in this article we only consider the case where all sources are located at a single redshift to simplify the comparisons with numerical simulations and the dependence on the source redshift). Then, using a flat-sky approximation, which is valid for small angles below a few degrees (Valageas et al. 2011), we define its 2D Fourier transform through

$\kappa(\boldsymbol{\theta})=\int \mathrm{d} \boldsymbol{\ell} \mathrm{e}^{\mathrm{i} \boldsymbol{\ell} \cdot \boldsymbol{\theta}} \tilde{\kappa}(\boldsymbol{\ell})$.

As in Paper I, we define the 2D convergence power spectrum and bispectrum as

$\left\langle\tilde{\kappa}\left(\boldsymbol{\ell}_{1}\right) \tilde{\kappa}\left(\boldsymbol{\ell}_{2}\right)\right\rangle=\delta_{D}\left(\boldsymbol{\ell}_{1}+\boldsymbol{\ell}_{2}\right) P_{\kappa}\left(\ell_{1}\right)$,

and

$\left\langle\tilde{\kappa}\left(\boldsymbol{\ell}_{1}\right) \tilde{\kappa}\left(\boldsymbol{\ell}_{2}\right) \tilde{\kappa}\left(\boldsymbol{\ell}_{3}\right)\right\rangle=\delta_{D}\left(\boldsymbol{\ell}_{1}+\boldsymbol{\ell}_{2}+\boldsymbol{\ell}_{3}\right) B_{\kappa}\left(\ell_{1}, \ell_{2}, \ell_{3}\right)$.

From Eq. (1) one obtains at once from Limber's approximation (Limber 1953; Kaiser 1992; Bartelmann \& Schneider 2001; Munshi et al. 2008)

$P_{\kappa}(\ell)=2 \pi \int_{0}^{\chi_{\mathrm{s}}} \mathrm{d} \chi \frac{w^{2}}{\mathcal{D}^{2}} P(\ell / \mathcal{D} ; z)$,

$B_{K}\left(\ell_{1}, \ell_{2}, \ell_{3}\right)=(2 \pi)^{2} \int_{0}^{\chi_{\mathrm{s}}} \mathrm{d} \chi \frac{w^{3}}{\mathcal{D}^{4}} B\left(\ell_{1} / \mathcal{D}, \ell_{2} / \mathcal{D}, \ell_{3} / \mathcal{D} ; z\right)$,

where $P(k ; z)$ and $B\left(k_{1}, k_{2}, k_{3} ; z\right)$ are the $3 \mathrm{D}$ power spectrum and bispectrum of the matter density contrast at redshift $z$. As described in Paper I, this provides the weak-lensing convergence power spectrum and bispectrum from the model we developed in Valageas \& Nishimichi (2011a,b) for the 3D power spectrum and bispectrum through a simple integration over the radial coordinate up to the source plane.

\subsection{Configuration-space statistics}

We focus here on configuration-space weak-lensing statistics, which may be more convenient than Fourier-space quantities for practical purposes because of complex survey geometries. Indeed, observations of the shear field are made in configuration space, by measuring large-scale correlations of galaxy ellipticities, and going to Fourier space (with further operations that are not local in real space) can be difficult because galaxy surveys do not cover the whole sky and shear maps can show irregular boundaries and internal holes due to observational constraints.

In particular, we consider the two-point and three-point correlation functions of the weak-lensing convergence, defined as

$\left\langle\kappa\left(\boldsymbol{\theta}_{1}\right) \kappa\left(\boldsymbol{\theta}_{2}\right)\right\rangle=\xi_{\kappa}\left(\left|\boldsymbol{\theta}_{2}-\boldsymbol{\theta}_{1}\right|\right)$,

$\left\langle\kappa\left(\boldsymbol{\theta}_{1}\right) \kappa\left(\boldsymbol{\theta}_{2}\right) \kappa\left(\boldsymbol{\theta}_{3}\right)\right\rangle=\zeta_{\kappa}\left(\left|\boldsymbol{\theta}_{2}-\boldsymbol{\theta}_{3}\right|,\left|\boldsymbol{\theta}_{3}-\boldsymbol{\theta}_{1}\right|,\left|\boldsymbol{\theta}_{1}-\boldsymbol{\theta}_{2}\right|\right)$,

where we used statistical homogeneity and isotropy (hence $\zeta_{k}$ only depends on the lengths of the three sides of the triangle defined by the summits $\left\{\boldsymbol{\theta}_{1}, \boldsymbol{\theta}_{2}, \boldsymbol{\theta}_{3}\right\}$ and the bispectrum (7) only depends on the three lengths $\left\{\ell_{1}, \ell_{2}, \ell_{3}\right\}$ ).

These real-space correlations can be expressed in terms of the Fourier-space power spectrum and bispectrum as

$\xi_{\kappa}(\theta)=2 \pi \int_{0}^{\infty} \mathrm{d} \ell \ell P_{K}(\ell) J_{0}(\ell \theta)$

where $\theta=\left|\boldsymbol{\theta}_{2}-\boldsymbol{\theta}_{1}\right|$ is the pair angular distance as in Eq. (8), and $\zeta_{\kappa}\left(v_{1}, v_{2}, v_{3}\right)=\frac{\pi}{v_{1}^{2} v_{2}^{2}} \int_{0}^{\infty} \mathrm{d} \ell \ell^{3} \int_{0}^{\pi / 2} \mathrm{~d} \theta \sin (2 \theta)$
$\times \int_{0}^{2 \pi} \mathrm{d} \varphi B_{\kappa}\left(\ell_{1}, \ell_{2}, \ell_{3}\right) J_{0}\left(\ell \sqrt{1+\sin (2 \theta) \cos \left(\varphi+\alpha_{3}\right)}\right)$,

where $\left\{v_{1}, v_{2}, v_{3}\right\}$ are the lengths of the three sides of the triangle $\left\{\boldsymbol{\theta}_{1}, \boldsymbol{\theta}_{2}, \boldsymbol{\theta}_{3}\right\}$ as in Eq. (9), $\alpha_{3}$ is the inner angle at summit $\boldsymbol{\theta}_{3}$, we chose without loss of generality

$v_{1} \geq v_{2} \geq v_{3}, \quad \cos \alpha_{3}=\frac{v_{1}^{2}+v_{2}^{2}-v_{3}^{2}}{2 v_{1} v_{2}}$,

and we noted in Eq. (11) the multipoles

$\ell_{1}=\frac{\ell \cos \theta}{v_{2}}, \quad \ell_{2}=\frac{\ell \sin \theta}{v_{1}}, \quad \ell_{3}^{2}=\ell_{1}^{2}+\ell_{2}^{2}+2 \ell_{1} \ell_{2} \cos \varphi$.

In addition to the three-point correlation $\zeta_{K}$ of the convergence, it can be useful to consider the three-point correlation of the cosmic shear $\gamma$. Because the latter is a spin-2 quantity, one is led to consider several three-point shear correlations (or "natural components"), depending on the choice of the reference direction (or of the projection procedure of the cosmic shear vectors), see Schneider \& Lombardi (2003); Schneider et al. (2005). However, they can all be written as integrals over the convergence bispectrum, such as Eq. (11), or integrals over the convergence three-point correlation (9) (Shi et al. 2011). Therefore, although we only consider the convergence three-point correlation (9) in this paper, we can expect a similar level of agreement between our analytical model and simulations for these other three-point correlations (in addition we also consider the thirdorder moment of the aperture-mass, which can be related to both the convergence and the cosmic shear). 
It is also common practice to study smoothed averages $X_{\mathrm{s}}$ of the convergence or shear field, defined by their filtering window $W_{\theta_{\mathrm{s}}}^{X_{\mathrm{s}}}(\boldsymbol{\theta})$ through

$X_{\mathrm{s}}=\int \frac{\mathrm{d} \boldsymbol{\theta}}{\pi \theta_{\mathrm{s}}^{2}} \kappa(\boldsymbol{\theta}) W_{\theta_{\mathrm{s}}}^{X_{\mathrm{s}}}(\boldsymbol{\theta})$.

For instance, the smoothed convergence $\kappa_{\mathrm{S}}$ is defined by a tophat filtering,

$W_{\theta_{\mathrm{s}}}^{K_{\mathrm{s}}}(\boldsymbol{\theta})=1$ if $|\boldsymbol{\theta}|<\theta_{\mathrm{s}}$ and zero otherwise,

while the "aperture-mass" $M_{\text {ap }}$ is defined by a compensated filter (Schneider 1996; Van Waerbeke et al. 2001), such as

$W_{\theta_{\mathrm{s}}}^{M_{\mathrm{ap}}}(\boldsymbol{\theta})=3\left(1-\frac{\theta^{2}}{\theta_{\mathrm{s}}^{2}}\right)\left(1-3 \frac{\theta^{2}}{\theta_{\mathrm{s}}^{2}}\right)$ if $|\boldsymbol{\theta}|<\theta_{\mathrm{s}}$,

and $W_{\theta_{\mathrm{s}}}^{M_{\mathrm{ap}}}(\boldsymbol{\theta})=0$ if $|\boldsymbol{\theta}|>\theta_{\mathrm{s}}$. This also reads in Fourier space as

$X_{\mathrm{s}}=\int \mathrm{d} \boldsymbol{\ell} \tilde{\kappa}(\boldsymbol{\ell}) \tilde{W}_{\theta_{\mathrm{s}}}^{X_{\mathrm{s}}}\left(\boldsymbol{\ell} \theta_{\mathrm{s}}\right)$

with

$\tilde{W}_{\theta_{\mathrm{s}}}^{X_{\mathrm{s}}}\left(\boldsymbol{\ell} \theta_{\mathrm{s}}\right)=\int \frac{\mathrm{d} \boldsymbol{\theta}}{\pi \theta_{\mathrm{s}}^{2}} \mathrm{e}^{\mathrm{i} \boldsymbol{\ell} \cdot \boldsymbol{\theta}} W_{\theta_{\mathrm{s}}}^{X_{\mathrm{s}}}(\boldsymbol{\theta})$.

In particular, we have

$\tilde{W}_{\theta_{\mathrm{s}}}^{K_{\mathrm{s}}}\left(\ell \theta_{\mathrm{s}}\right)=2 \frac{J_{1}\left(\ell \theta_{\mathrm{s}}\right)}{\ell \theta_{\mathrm{s}}}, \quad \tilde{W}_{\theta_{\mathrm{s}}}^{M_{\mathrm{ap}}}\left(\ell \theta_{\mathrm{s}}\right)=24 \frac{J_{4}\left(\ell \theta_{\mathrm{s}}\right)}{\left(\ell \theta_{\mathrm{s}}\right)^{2}}$.

We mainly focus here on one-point moments of $\kappa_{\mathrm{s}}$ and $M_{\mathrm{ap}}$, that is $\left\langle X_{\mathrm{s}}^{p}\right\rangle$, and we do not consider multi-point statistics such as $\left\langle X_{\mathrm{s}}\left(\boldsymbol{\theta}_{1} ; \theta_{\mathrm{s} 1}\right) \ldots X_{\mathrm{s}}\left(\boldsymbol{\theta}_{p} ; \theta_{\mathrm{s} p}\right)\right\rangle$ associated with $p$ windows centered on $p$ different directions and with $p$ different angular radii. However, we will check the validity of our model for multi-scale statistics, that is, for windows of different sizes centered on the same direction, in Sect. 8.

In the simpler case of one-point moments $\left\langle X_{\mathrm{s}}^{p}\right\rangle$ the variance reads as

$\left\langle X_{\mathrm{s}}^{2}\right\rangle=2 \pi \int_{0}^{\infty} \mathrm{d} \ell \ell P_{\kappa}(\ell) \tilde{W}_{\theta_{\mathrm{s}}}^{X_{\mathrm{s}}}\left(\ell \theta_{\mathrm{s}}\right)^{2}$,

while the third-order moment reads as

$$
\begin{aligned}
\left\langle X_{\mathrm{s}}^{3}\right\rangle & =24 \pi \int_{0}^{\infty} \mathrm{d} \ell_{1} \ell_{1} \int_{\ell_{1} / 2}^{\ell_{1}} \mathrm{~d} \ell_{2} \ell_{2} \int_{0}^{\arccos \left[\ell_{1} /\left(2 \ell_{2}\right)\right]} \mathrm{d} \varphi \\
& \times B_{\kappa}\left(\ell_{1}, \ell_{2}, \ell_{3}\right) \tilde{W}_{\theta_{\mathrm{s}}}^{X_{\mathrm{s}}}\left(\ell_{1} \theta_{\mathrm{s}}\right) \tilde{W}_{\theta_{\mathrm{s}}}^{X_{\mathrm{s}}}\left(\ell_{2} \theta_{\mathrm{s}}\right) \tilde{W}_{\theta_{\mathrm{s}}}^{X_{\mathrm{s}}}\left(\ell_{3} \theta_{\mathrm{s}}\right),
\end{aligned}
$$

where we used the symmetries of the bispectrum and we noted $\ell_{3}^{2}=\ell_{1}^{2}+\ell_{2}^{2}-2 \ell_{1} \ell_{2} \cos \varphi$.

In practice, to avoid the numerous oscillations and changes of sign brought by the Fourier-space filters $\tilde{W}_{\theta_{\mathrm{s}}}^{X_{\mathrm{s}}}$ given in Eq. (19), we found it convenient to express the third-order moment (21) in terms of the real-space three-point correlation (9), although this yields a five-dimensional integral instead of the threedimensional integral (21),

$$
\begin{aligned}
\left\langle X_{\mathrm{s}}^{3}\right\rangle= & \frac{24 \pi}{\left(\pi \theta_{\mathrm{s}}^{2}\right)^{3}} \int_{0}^{\theta_{\mathrm{s}}} \mathrm{d} \theta_{1} \theta_{1} W_{\theta_{\mathrm{s}}}^{X_{\mathrm{s}}}\left(\theta_{1}\right) \int_{0}^{\theta_{1}} \mathrm{~d} \theta_{2} \theta_{2} W_{\theta_{\mathrm{s}}}^{X_{\mathrm{s}}}\left(\theta_{2}\right) \\
& \times \int_{0}^{\theta_{2}} \mathrm{~d} \theta_{3} \theta_{3} W_{\theta_{\mathrm{s}}}^{X_{\mathrm{s}}}\left(\theta_{3}\right) \int_{0}^{\pi} \mathrm{d} \varphi_{2} \int_{0}^{2 \pi} \mathrm{d} \varphi_{3} \zeta_{\kappa}\left(v_{1}, v_{2}, v_{3}\right),
\end{aligned}
$$

with

$$
\begin{aligned}
& v_{1}^{2}=\left(\theta_{3} \cos \varphi_{3}-\theta_{2} \cos \varphi_{2}\right)^{2}+\left(\theta_{3} \sin \varphi_{3}-\theta_{2} \sin \varphi_{2}\right)^{2} \\
& v_{2}^{2}=\left(\theta_{3} \cos \varphi_{3}-\theta_{1}\right)^{2}+\theta_{3}^{2} \sin ^{2} \varphi_{3}, \\
& v_{3}^{2}=\left(\theta_{2} \cos \varphi_{2}-\theta_{1}\right)^{2}+\theta_{2}^{2} \sin ^{2} \varphi_{2}
\end{aligned}
$$

\section{Numerical simulations}

We performed the ray-tracing simulations through highresolution $N$-body simulations of cosmological structure formation (Jain et al. 2000; Hamana et al. 2001; Sato et al. 2009; Takahashi et al. 2011) to obtain accurate predictions of the configuration statistics for weak lensing. To run the $N$-body simulations, we used a modified version of the Gadget-2 code (Springel 2005) and employed $256^{3}$ particles for each simulation. The ray-tracing simulations were constructed from $2 \times 200$ realizations of $N$-body simulations with cubic 240 and $480 h^{-1} \mathrm{Mpc}$ on a side, respectively, to cover a light cone of angular size $5^{\circ} \times 5^{\circ}$ (see Fig. 1 in Sato et al. 2009). For our fiducial cosmology, we adopted the standard $\Lambda$ CDM cosmology with matter fraction $\Omega_{\mathrm{m}}=0.238$, baryon fraction $\Omega_{\mathrm{b}}=0.0416$, dark energy fraction $\Omega_{\mathrm{de}}=0.762$ with the equation of state parameters $w_{0}=-1$ and $w_{a}=0$, spectral index $n_{\mathrm{s}}=0.958$, normalization $A_{\mathrm{s}}=2.35 \times 10^{-9}$, and Hubble parameter $h=0.732$, which are consistent with the WMAP three-year results (Spergel et al. 2007). This fiducial cosmology gives the normalization $\sigma_{8}=0.759$ for the variance of the linear density fluctuations in a sphere of radius $8 h^{-1} \mathrm{Mpc}$. We considered source redshifts at either $z_{\mathrm{s}}=0.6,1.0$, or 1.5 . Using ray-tracing simulations we generated 1000 realizations of convergence maps for each source redshift.

In addition to the fiducial cosmology case, we performed ray-tracing simulations for several slightly different cosmologies. We varied each of the following cosmological parameters: $A_{\mathrm{s}}, n_{\mathrm{s}}$, the cold dark matter density $\Omega_{\mathrm{c}} h^{2}, \Omega_{\mathrm{de}}$, and $w_{0}$ by $\pm 10 \%$, respectively. Therefore $h, \Omega_{\mathrm{m}}$, and $\Omega_{\mathrm{b}}$ are dependent parameters, because we assumed that the Universe is flat and the baryon density $\Omega_{\mathrm{b}} h^{2}$ is fixed. For each of these ten different cosmologies, we obtain 40 realizations of convergence fields for each of the three source redshifts. Details of the methods used for the ray-tracing simulations can be found in Sato et al. (2009). All convergence maps used in this paper are the same as those used in Paper I.

In Sects. 4-6, we use the maps for the fiducial cosmology, while in Sect. 7 we use those for the varied cosmologies to investigate the robustness of our model. In Sect. 7 we show the results for six cases, varying $n_{\mathrm{s}}, \Omega_{\mathrm{c}} h^{2}$, and $w_{0}$ by $\pm 10 \%$. The exact values for these cosmological parameters are listed in Table A.1 in Paper I.

\section{Lensing two-point functions}

We now compare our results for weak-lensing two-point functions with numerical simulations. As in Paper I, we also considered the predictions obtained from the popular "halo-fit" fitting function for the 3D density power spectrum given in Smith et al. (2003), to estimate the advantages of more systematic approaches like ours.

We show our results for the convergence two-point correlation $\xi_{\kappa}(\theta)$, the variance of the smoothed convergence $\left\langle\kappa_{\mathrm{s}}^{2}\right\rangle$, and the variance of the aperture mass $\left\langle M_{\mathrm{ap}}^{2}\right\rangle$ in Fig. 1. The numerical error bars increase on large scales because of the finite size of the simulation box. On small scales the numerical error is dominated by systematic effects, because of the finite resolution, which leads to an underestimation of the small-scale power. This underestimation was clearly apparent for the power spectrum $P_{K}(\ell)$ shown in Paper I and can also be seen (especially at low redshift) for the variance of the aperture mass, which involves a compensated filter and probes a narrow range of scales (Schneider 1996). For each source redshift we estimated the angular scale down to 

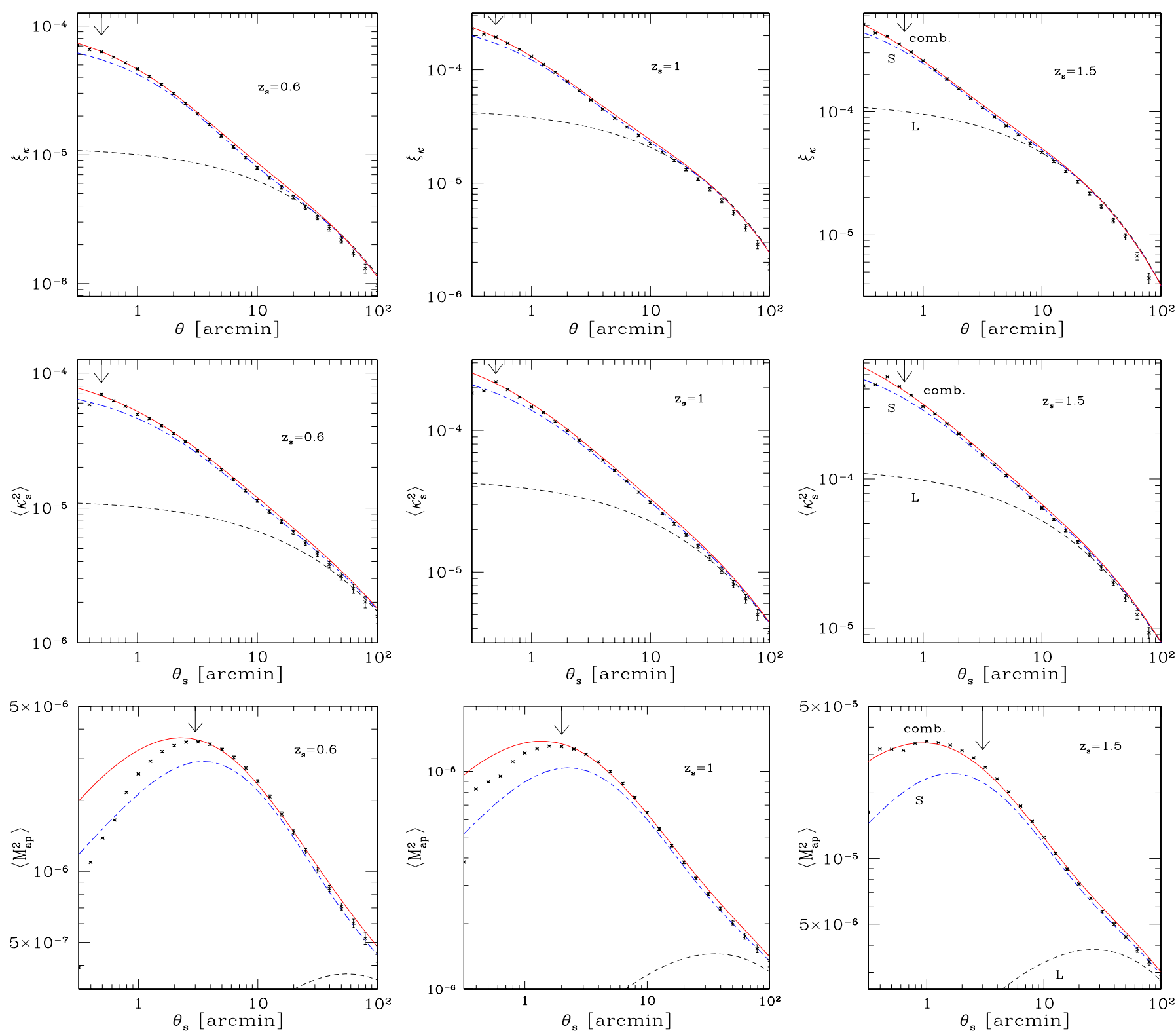

Fig. 1. Upper row: convergence two-point correlation function for sources at redshifts $z_{\mathrm{s}}=0.6,1$, and 1.5 , as a function of the angular pair separation $\boldsymbol{\theta}$. The points are the results from numerical simulations with $3-\sigma$ error bars. The low black dashed line "L" is the linear correlation, the middle blue dash-dotted line "S" is the result from the "halo-fit" of Smith et al. (2003), and the upper red solid line "comb." is the result from our model, which combines one-loop perturbation theory with a halo model. The vertical arrow shows the scale down to which the simulation result is valid within 5\%. Middle row: variance of the smoothed convergence for the same cases, as a function of the smoothing angle $\theta_{\mathrm{s}}$. Lower row: variance of the aperture mass for the same cases, as a function of the smoothing angle $\theta_{\mathrm{s}}$.

which the simulations have an accuracy of better than $5 \%$ by comparing with higher-resolution simulations (with $512^{3}$ particles instead of $256^{3}$ ). This scale is shown by the vertical arrow in Fig. 1 and we can check that our model indeed agrees with the numerical simulations down to this angular scale.

The two-point correlation and the smoothed convergence are not as sensitive to this low-resolution effect because they involve uncompensated filters, $\tilde{W}(0) \neq 0$, which implies that at a given smoothing angular scale $\theta_{\mathrm{s}}$ they receive greater contributions from larger scales (which are unaffected by the numerical resolution) than the aperture mass.

For the same reason, $\xi_{k}(\theta)$ and $\left\langle\kappa_{\mathrm{s}}^{2}\right\rangle$ remain adequately described by linear theory down to $\sim 10 \operatorname{arcmin}$, whereas $\left\langle M_{\mathrm{ap}}^{2}\right\rangle$ already shows significant deviations at $\sim 100$ arcmin. This also explains why the predictions from our model and the "halo-fit" are closer for the first two statistics than for $M_{\mathrm{ap}}$. In agreement with our results for the convergence power spectrum (Paper I) and previous works (White \& Vale 2004; Hilbert et al. 2009; Sato et al. 2009; Semboloni et al. 2011), the "halo-fit" formula underestimates the power on small scales. The discrepancy is again larger for the aperture mass. As in Paper I, our model provides a more accurate match to the numerical simulations down to $\sim 1$ arcmin. On smaller scales the results from simulations show a fast drop (especially for $M_{\text {ap }}$ ) that is not physical but due to the finite resolution. This prevents an accurate comparison with our predictions. However, since our model is built from a physical halo model and has been tested for the 3D density field down to highly nonlinear scales with higher-resolution simulations (Valageas \& Nishimichi 2011a), it should be more reliable than the numerical results shown in Fig. 1 below $\sim 1$ arcmin. This 
shows one advantage of analytic (or semi-analytic) approaches as compared with numerical simulations: they can provide realistic predictions on a wider range of scales.

\section{Lensing three-point functions}

We now compare our analytical results with numerical simulations for three-point functions. As in Paper I, we also considered the predictions obtained from the following three simple models, which have been used in some previous works. (We did not consider the scale transformation introduced in Pan et al. (2007) because we have already shown in Paper I that it does not provide a sufficiently accurate model for the convergence bispectrum. We have checked that we obtain similar results for $\left\langle\kappa_{\mathrm{s}}^{3}\right\rangle$ and $\left\langle M_{\mathrm{ap}}^{3}\right\rangle$.)

The first model, "tree $\mathrm{L}_{\mathrm{L}}$, is the lowest-order ("tree-order") prediction from standard perturbation theory, which reads for the 3D bispectrum (Bernardeau et al. 2002)

$B_{\text {tree }_{\mathrm{L}}}\left(k_{1}, k_{2}, k_{3}\right)=2 F_{2}\left(k_{1}, k_{2}, \mu_{12}\right) P_{L}\left(k_{1}\right) P_{L}\left(k_{2}\right)+2$ cyc.

where $\mu_{12}=\left(\boldsymbol{k}_{1} \cdot \boldsymbol{k}_{2}\right) /\left(k_{1} k_{2}\right)$ and

$F_{2}\left(k_{1}, k_{2}, \mu_{12}\right)=\frac{5}{7}+\frac{1}{2}\left(\frac{k_{1}}{k_{2}}+\frac{k_{2}}{k_{1}}\right) \mu_{12}+\frac{2}{7} \mu_{12}^{2}$.

The second model, "tree $\mathrm{NL}$ ", is given by Eq. (27) where we replace the linear 3D power $P_{L}(k)$ by the nonlinear power $P_{S}(k)$ from Smith et al. (2003). The third model, " $F_{2, \mathrm{NL}}$ ", makes the additional modification to replace the kernel $F_{2}$ by an effective kernel $F_{2, \mathrm{NL}}$ that interpolates from the large-scale perturbative result (28) to a small-scale ansatz where the angular dependence on $\mu_{12}$ vanishes, using the fitting formula from Scoccimarro \& Couchman (2001). We considered two variants, using either the "halo-fit" nonlinear power spectrum $P_{S}(k)$ from Smith et al. (2003) or the nonlinear power spectrum $P_{\text {tang }}(k)$ of our model, see Paper I and Valageas \& Nishimichi (2011b).

We show our results for the convergence three-point correlation function $\zeta_{\kappa}$ (for equilateral triangles) and for the third-order moments $\left\langle\kappa_{\mathrm{s}}^{3}\right\rangle$ and $\left\langle M_{\mathrm{ap}}^{3}\right\rangle$ in Fig. 2. In agreement with the secondorder statistics shown in Fig. 1, the lowest-order perturbation theory prediction, "tree ${ }_{\mathrm{L}}$, , remains valid down to smaller angular scales for $\zeta_{\kappa}$ and $\kappa_{\mathrm{S}}$ than for $M_{\mathrm{ap}}$ because the former involve uncompensated filters instead of a compensated filter.

The simulations slightly underestimate the power on moderate and large angular scales because of the finite size of the simulation box $\left(240 h^{-1}\right.$ and $480 h^{-1} \mathrm{Mpc}$, which corresponds to 343 and 686 arcmin at $z=1$ ), which cuts contributions from longer wavelengths. This discrepancy is not due to the analytic model because on the largest angular scales we can check that all theoretical predictions converge on the linear theory (as they must for CDM power spectra) while predicting somewhat more power than measured in the simulations (see the first row in Fig. 2). Therefore, this mismatch is not caused by higher-order perturbative corrections (e.g., two-loop terms), which are even smaller than the one-loop contributions that we included in our model. In agreement with this explanation, the discrepancy is smaller for $M_{\text {ap }}$ than for $\zeta_{\kappa}$ and $\kappa$ because, for a given smoothing radius $\theta_{\mathrm{s}}, M_{\text {ap }}$ is less sensitive to larger scales thanks to its compensated filter $\tilde{W}_{\theta_{\mathrm{s}}}^{M_{\text {ap }}}$. This shows that analytical models, such as the one we propose here, are competitive with numerical simulations if one needs to describe a broad range of scales.

In agreement with the results obtained in Paper I for the convergence bispectrum, using the nonlinear power within the "treelevel" expression (27) significantly increases the third-order moments on smaller scales and improves the general shape but is not sufficient to bridge the gap with the simulations. Modifying the kernel $F_{2}$ by using the fitting formula of Scoccimarro \& Couchman (2001) improves the predictions even more, especially when we use the 3D nonlinear power spectrum given by our model, which was shown earlier to be reasonably accurate (see Fig. 1; Paper I; Valageas \& Nishimichi 2011b). Indeed, as for the variance, the "halo-fit" power spectrum of Smith et al. (2003) yields too little power on small scales, in agreement with previous works (Semboloni et al. 2011). However, this approach still underestimates the weak-lensing signal.

The best agreement with the numerical simulations is provided by our model. As was seen for the convergence bispectrum in Paper I, it is interesting to note again the good match on the transition scales, $\theta_{\mathrm{s}} \sim 5$ arcmin, which a priori are the most difficult to reproduce since they are at the limit of validity of both perturbative approaches (which break down at shell crossing) and halo models (which assume virialized halos). On small angular scales we again predict more power than is measured in the simulations, but like for the second-order moments this is at least partly caused by the lack of small-scale power in the simulations because of the finite resolution. Thus, we again plot in Fig. 2 the vertical arrows that were plotted in Fig. 1. Since the bispectrum typically scales as the square of the power spectrum, this should roughly correspond to an accuracy threshold of about $10 \%$ for the simulations. We can check that our model agrees with the numerical results down to this angular scale. As in Fig. $1, M_{\mathrm{ap}}$ is much more sensitive than $\zeta_{K}$ and $\kappa_{\mathrm{S}}$ to this finiteresolution effect.

\section{Relative importance of the different contributions}

As in Paper I, we have seen in the previous section that our model, which is based on a combination of perturbation theories and halo models, provides a good match to numerical simulations. Therefore, it can be used to predict weak-lensing statistics for a variety of cosmologies, which is an important goal for observational and practical purposes. A second use of our approach is to compare the different contributions that eventually add up to the signal that can be measured in weak-lensing surveys. Thus, we can distinguish the various perturbative terms as well as the nonperturbative contributions associated for instance with onehalo or two-halo terms. This is a second advantage of these models, as compared with fitting formulas or direct ray-tracing simulations. This enables a deeper understanding of which properties of the matter distribution, and of the overall cosmological setting, can be probed by a given gravitational lensing measure. It can also help to estimate the accuracy that can be aimed at in weak-lensing statistics as a function of scales, because different contributions suffer from different theoretical uncertainties.

\subsection{Two-point statistics}

We plot our results for $\xi_{\kappa},\left\langle\kappa_{\mathrm{s}}^{2}\right\rangle$, and $\left\langle M_{\mathrm{ap}}^{2}\right\rangle$ in Fig. 3 at redshift $z_{\mathrm{s}}=1$. In addition to the full model prediction that was already shown in Fig. 1, we show the underlying 2-halo and 1-halo contributions. Because of their uncompensated filters, which make statistics at a given angular scale receive contributions from 3D fluctuations on a wide range of scales, taking the one-loop perturbative term into account only yields a small increase of $\xi_{\kappa}$ and $\left\langle\kappa_{\mathrm{s}}^{2}\right\rangle$ over a wide range of angular scales, as compared with the linear prediction. In contrast, for $\left\langle M_{\mathrm{ap}}^{2}\right\rangle$ this one-loop contribution peaks on a narrow range of angular scales around 40 arcmin and has a significant impact that improves the match 

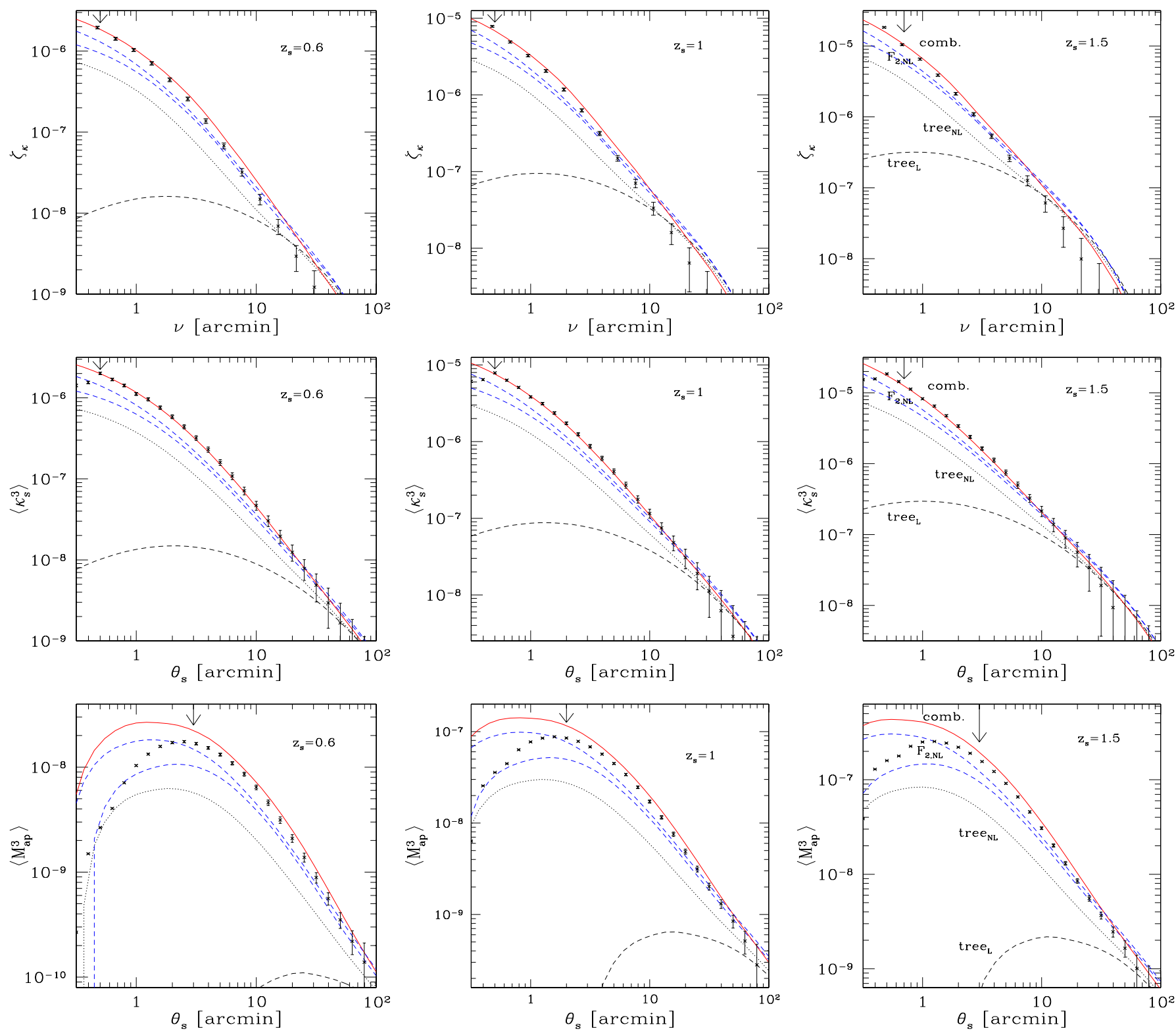

Fig. 2. Upper row: convergence three-point correlation function for equilateral triangles, as a function of the triangle side $v$, for sources at redshifts $z_{\mathrm{s}}=0.6,1$, and 1.5 . The points are the results from numerical simulations with $3-\sigma$ error bars. The low black dashed line "tree ${ }_{\mathrm{L}}$ ", the black dotted line "tree $\mathrm{NL}$ " and the two blue dashed lines " $F_{2, \mathrm{NL}}$ " are obtained from the ansatz (27), using either the linear 3D power spectrum, or the "halo-fit" power, or an effective kernel $F_{2, \mathrm{NL}}$ with the "halo-fit" power (lower curve) or the power from our model (upper curve). The red solid line "comb." is our combined model, described in Paper I. The vertical arrows are at the same angular scale as in Fig. 1. Middle row: third-order moment of the smoothed convergence, as a function of the smoothing angle $\theta_{\mathrm{s}}$. Lower row: third-order moment of the aperture mass, as a function of the smoothing angle $\theta_{\mathrm{s}}$.

to the numerical results. On small scales the two-halo contribution decreases close to the linear prediction thanks to the partial resummation of higher perturbative orders. As explained in Valageas \& Nishimichi (2011a) and Paper I, this is a useful improvement over the standard one-loop perturbation theory because it ensures that the two-halo term does not give significant contributions on very small scales, in agreement with physical expectations.

Then, these two-point weak-lensing quantities become dominated on small scales by the one-halo term but like for the 3D and 2D power spectra, there remains a significant intermediate range. Again, our model provides a satisfactory interpolation on these scales, but it would be interesting to build a more systematic procedure, for instance by including higher orders of perturbation theory or by building a more refined matching between the two-halo and one-halo regimes. In any case, Fig. 3 clearly shows how $\xi_{\kappa},\left\langle\kappa_{\mathrm{s}}^{2}\right\rangle$, and $\left\langle M_{\mathrm{ap}}^{2}\right\rangle$ depend on large-scale perturbative density fluctuations or on small-scale halo properties, as the angular scale varies.

We checked that we obtain similar results at redshifts $z_{\mathrm{s}}=$ 0.6 and 1.5 .

\subsection{Three-point statistics}

We now study the various contributions to the lensing three-point functions, associated with the thee-halo, two-halo, and one-halo terms.

We plot our results for $\zeta_{\kappa}$ (for equilateral configurations), $\left\langle\kappa_{\mathrm{s}}^{3}\right\rangle$, and $\left\langle M_{\mathrm{ap}}^{3}\right\rangle$ in Fig. 4, at redshift $z_{\mathrm{s}}=1$. As in Paper I, the three-halo term is identified with the perturbative prediction and 
P. Valageas et al.: Modeling of weak-lensing statistics. II.
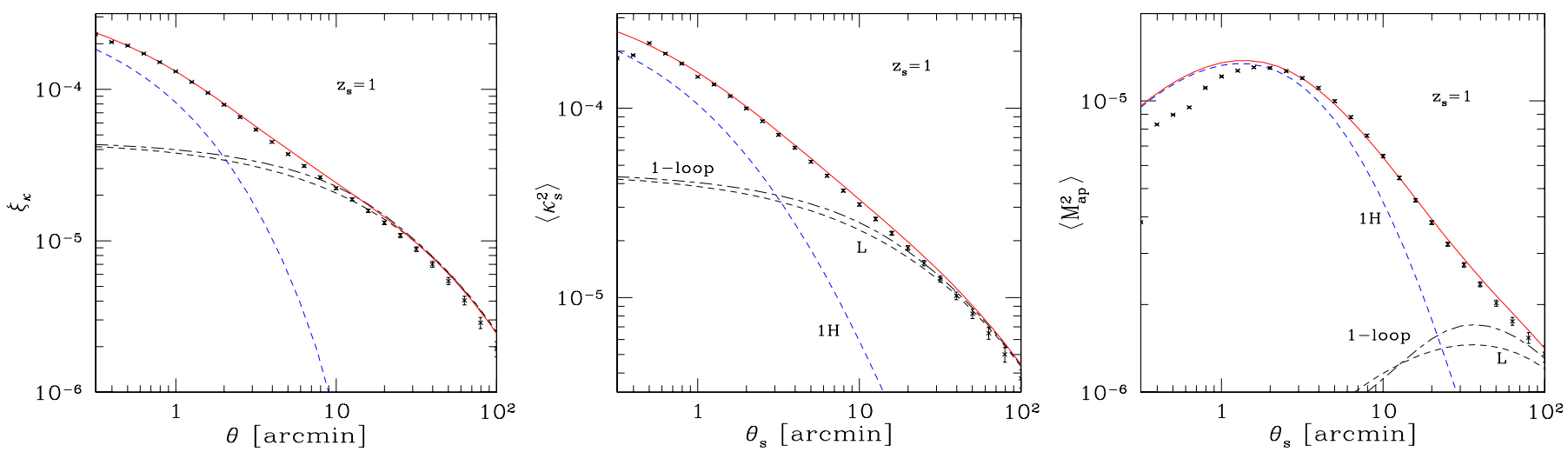

Fig. 3. Convergence two-point correlation function (left panel), smoothed convergence (middle panel), and aperture-mass (right panel) for sources at redshift $z_{\mathrm{s}}=1$. The points are the results from numerical simulations with $3-\sigma$ error bars. The low black dashed line "L" is the linear prediction, the middle black dash-dotted line "1-loop" is the two-halo contribution, for which we used a perturbative resummation that is complete up to one-loop order, the upper blue dashed line " $1 \mathrm{H}$ " is the one-halo contribution, and the red solid line is our full model, as in Fig. 1.
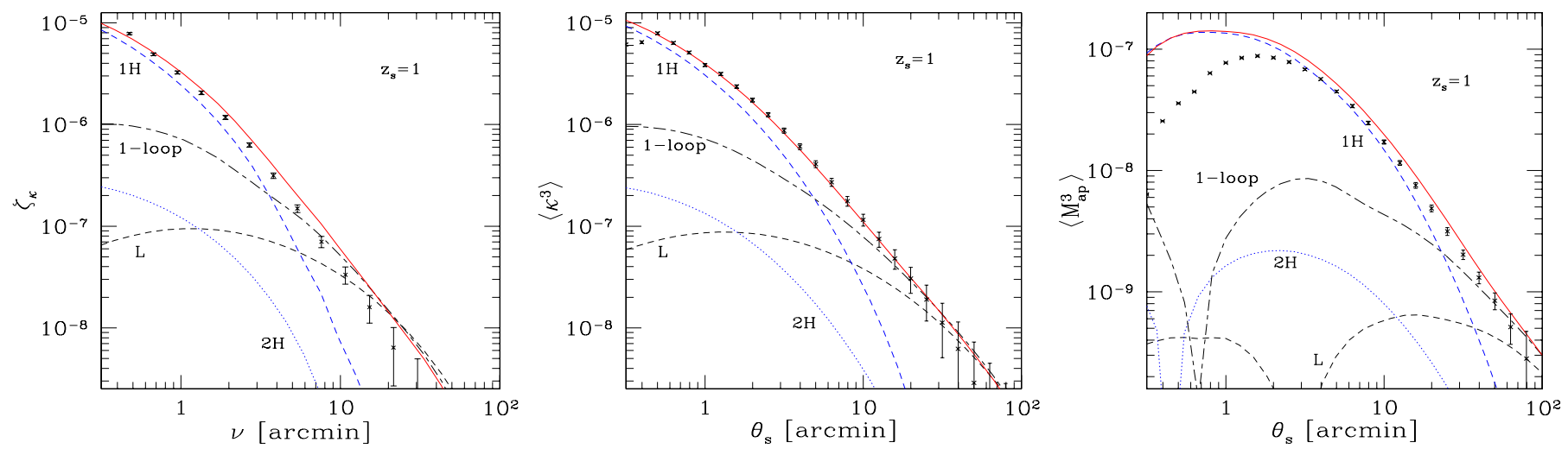

Fig. 4. Convergence three-point correlation $\zeta_{k}$ for equilateral configurations (left panel) and third-order moments $\left\langle\kappa_{\mathrm{s}}^{3}\right\rangle$ (middle panel) and $\left\langle M_{\mathrm{ap}}^{3}\right\rangle$ (right panel), for sources at redshift $z_{\mathrm{s}}=1$. The points are the results from numerical simulations with $3-\sigma$ error bars. The low black dashed line "L" is the lowest-order perturbative prediction (27), the "1-loop" dash-dotted black line is the prediction of one-loop standard perturbation theory, which is identified with our three-halo term, the blue dotted line " $2 \mathrm{H}$ " is the two-halo contribution and the upper blue dashed line " $1 \mathrm{H}$ " is the one-halo contribution. The red solid line is our full model.

we used the standard perturbation theory at one-loop order. Like for the 3D bispectrum (Valageas \& Nishimichi 2011b), and contrary to the power spectrum, this gives a contribution that becomes negligible on small scales, so that it is not necessary to use a resummation scheme or to add a non-perturbative cutoff to ensure a good small-scale behavior. On the other hand, contrary to the two-point statistics shown in Fig. 3, going to one-loop order now provides a great improvement over the tree-order result, even for $\zeta_{\kappa}$ and $\left\langle\kappa_{\mathrm{s}}^{3}\right\rangle$. This feature was already noticed for the 3D bispectrum (Sefusatti et al. 2010; Valageas \& Nishimichi 2011b) and the convergence bispectrum (Paper I). Thus, combining this one-loop perturbative contribution with the two-halo and onehalo terms is sufficient to obtain a good match to the simulations, from the quasilinear to the highly nonlinear scales. This suggests that higher orders of perturbation theory do not significantly contribute to the bispectrum and that we already have a reasonably successful model. The two-halo term is also subdominant on all scales (by a factor $\sim 10$ at least). This is a nice property since because it is a mixed term, which involves both large-scale halo correlations and internal halo structures, it may be more difficult to predict than the three-halo term (which is derived from systematic perturbation theories) and the one-halo term (which only depends on internal halo profiles and mass function). These various features were also observed for the 3D bispectrum (Valageas $\&$ Nishimichi 2011b) and the convergence bispectrum (Paper I).
Again, we checked that we obtain similar results at redshifts $z_{\mathrm{s}}=0.6$ and 1.5 .

\section{Dependence on cosmology}

In this section we check the robustness of our model when we vary the cosmological parameters. As in Paper I, we considered six alternative cosmologies, where $n_{\mathrm{s}}, \Omega_{\mathrm{c}} h^{2}$, and $w_{0}$ are modified by $\pm 10 \%$ with respect to the fiducial cosmology used in the previous sections. The values of the associated cosmological parameters are given in Table I in Appendix A of Paper I. We compare the predictions of our model with numerical simulations for these six alternative cosmologies in Figs. 5 and 6 for the two-point and three-point statistics at $z_{\mathrm{s}}=1$. To avoid overcrowding the figures we did not plot the error bars of the numerical simulations. Each pair $n_{\mathrm{s}}, \Omega_{\mathrm{c}} h^{2}$, and $w_{0}$ gives two curves that are roughly symmetric around the fiducial cosmology result, because we consider small deviations of $\pm 10 \%$. The deviations are largest for the $n_{\mathrm{s}}$ case, which changes the shape of the initial power spectrum as well as the normalization $\sigma_{8}$. These six cases roughly cover the range that is allowed by current data, and the $n_{\mathrm{s}}$ case is already somewhat beyond the observational bounds (Komatsu et al. 2011). Therefore, they provide a good check of the robustness of our model for realistic scenarios. 

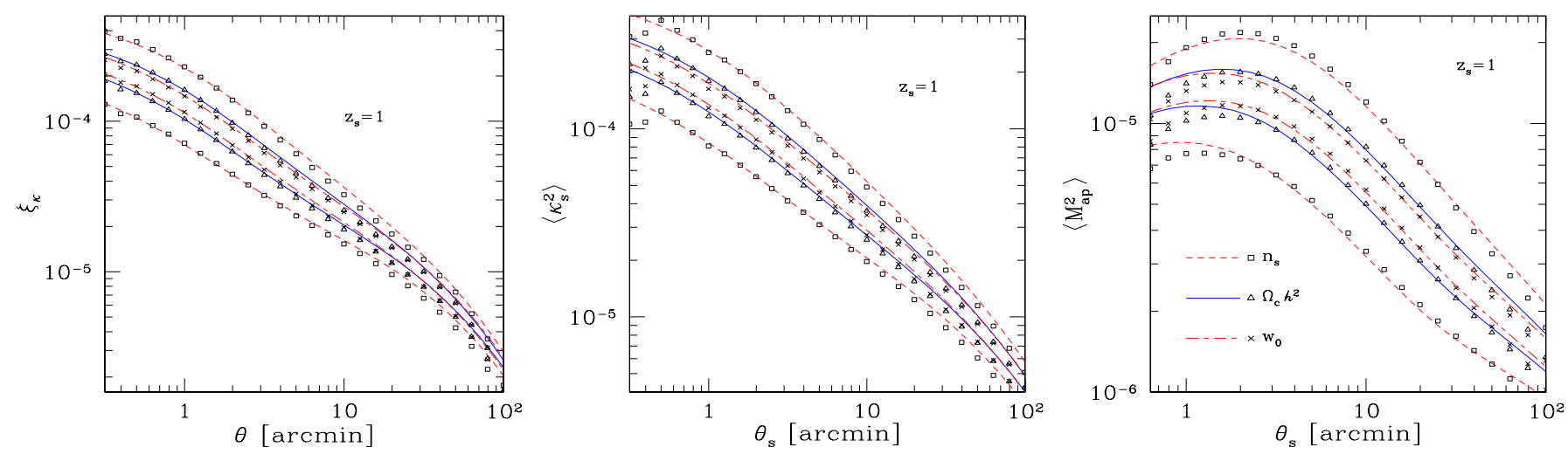

Fig. 5. Convergence two-point correlation function (left panel), smoothed convergence (middle panel), and aperture-mass (right panel) for sources at redshift $z_{\mathrm{s}}=1$ for six cosmologies. The points are the results from numerical simulations and the lines are the predictions of our model.
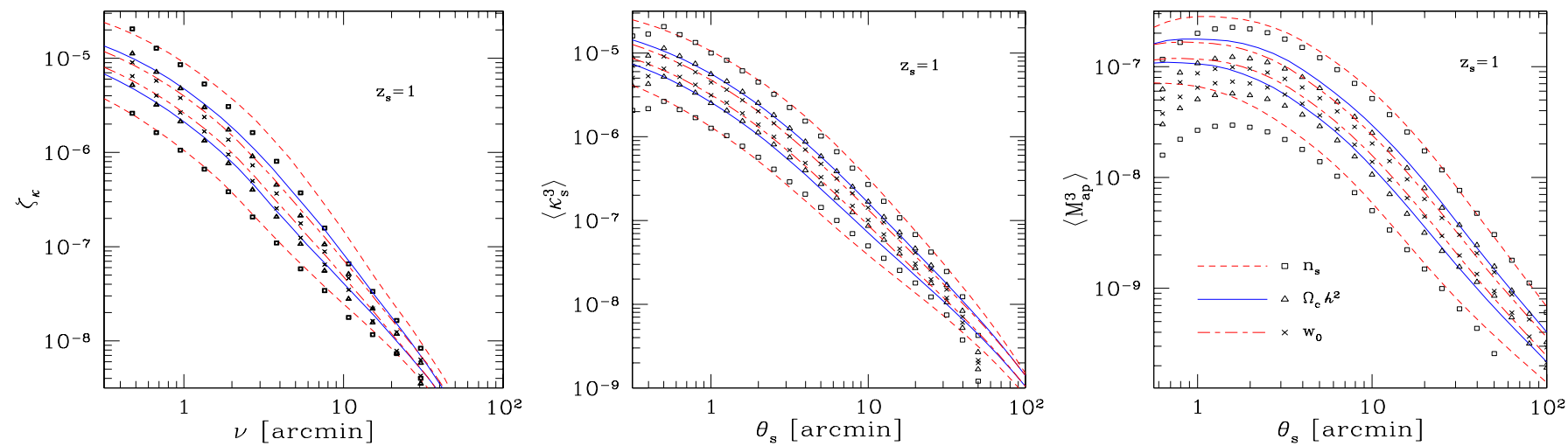

Fig. 6. Convergence three-point correlation $\zeta_{\kappa}$ for equilateral configurations (left panel) and third-order moments $\left\langle\kappa_{\mathrm{s}}^{3}\right\rangle\left(\right.$ middle panel) and $\left\langle M_{\mathrm{ap}}^{3}\right\rangle$ (right panel) for sources at redshift $z_{\mathrm{s}}=1$ for six cosmologies. The points are the results from numerical simulations and the lines are the predictions of our model.

Like for the Fourier-space statistics studied in Paper I, the dependence on cosmology of the two-point statistics is well reproduced by our model. For the three-point statistics it is not easy to make a very precise comparison because the numerical results show a greater level of noise and are sensitive to finite resolution and finite size effects. However, where the simulations are reliable, we also obtain a good match with our predictions. We obtained similar results for $z_{\mathrm{s}}=0.6$ and $z_{\mathrm{s}}=1.5$, as well as for other cosmologies where we vary $A_{\mathrm{s}}$ or $\Omega_{\mathrm{de}}$ by $\pm 10 \%$. This shows that our model and, more generally, models based on combinations of perturbation theory and halo models provide a good modeling of the matter distribution and of weak gravitational lensing effects and capture their dependence on cosmology. Moreover, Figs. 5 and 6 clearly show that this analytical modeling is competitive with current ray-tracing simulations, because it provides reliable predictions over a greater range of scales. In particular, Figs. 5 and 6 show that the accuracy of our model is sufficient to constrain $n_{\mathrm{s}}, \Omega_{\mathrm{c}} h^{2}$, and $w_{0}$ to better than $10 \%$.

\section{Multi-scale moments}

In the previous sections we considered single-scale moments, $\left\langle X_{\mathrm{s}}\left(\theta_{\mathrm{s}}\right)^{p}\right\rangle$, associated with one smoothing window $W_{\theta_{\mathrm{s}}}^{X_{\mathrm{s}}}$ with a single angular radius $\theta_{\mathrm{s}}$. One can also use multi-point statistics such as $\left\langle X_{\mathrm{s}}\left(\boldsymbol{\theta}_{1} ; \theta_{\mathrm{s} 1}\right) \ldots X_{\mathrm{s}}\left(\boldsymbol{\theta}_{p} ; \theta_{\mathrm{s} p}\right)\right\rangle$ associated with $p$ windows centered on $p$ different directions $\boldsymbol{\theta}_{i}$ and with $p$ different radii $\theta_{s i}$. In this section, we briefly check the validity of our model for the case of centered multi-scale moments. To do this, all quantities $X_{\mathrm{s} i}$ are centered on the same direction on the sky but we allow the angular radii $\theta_{s i}$ to be different. Then, Eqs. (20) and (23) generalize as

$$
\left\langle X_{\mathrm{s}}\left(\theta_{\mathrm{s} 1}\right) X_{\mathrm{s}}\left(\theta_{\mathrm{s} 2}\right)\right\rangle=2 \pi \int_{0}^{\infty} \mathrm{d} \ell \ell P_{\kappa}(\ell) \tilde{W}_{\theta_{\mathrm{s} 1}}^{X_{\mathrm{s}}}\left(\ell \theta_{\mathrm{s} 1}\right) \tilde{W}_{\theta_{\mathrm{s} 2}}^{X_{\mathrm{s}}}\left(\ell \theta_{\mathrm{s} 2}\right)
$$

and

$$
\begin{aligned}
& \left\langle X_{\mathrm{s}}\left(\theta_{\mathrm{s} 1}\right) X_{\mathrm{s}}\left(\theta_{\mathrm{s} 2}\right) X_{\mathrm{s}}\left(\theta_{\mathrm{s} 3}\right)\right\rangle=4 \pi \int_{0}^{\theta_{\mathrm{s} 1}} \frac{\mathrm{d} \theta_{1} \theta_{1}}{\pi \theta_{\mathrm{s} 1}^{2}} W_{\theta_{\mathrm{s} 1}}^{X_{\mathrm{s}}}\left(\theta_{1}\right) \\
& \quad \times \int_{0}^{\theta_{\mathrm{s} 2} \mathrm{~d} \theta_{2} \theta_{2}} \frac{\theta_{2}}{\pi \theta_{\mathrm{s} 2}^{2}} W_{\theta_{\mathrm{s} 2}}^{X_{\mathrm{s}}}\left(\theta_{2}\right) \int_{0}^{\theta_{\mathrm{s} 3} 3} \frac{\mathrm{d} \theta_{3} \theta_{3}}{\pi \theta_{\mathrm{s} 3}^{2}} W_{\theta_{\mathrm{s} 3}}^{X_{\mathrm{s}}}\left(\theta_{3}\right) \\
& \quad \times \int_{0}^{\pi} \mathrm{d} \varphi_{2} \int_{0}^{2 \pi} \mathrm{d} \varphi_{3} \zeta_{\kappa}\left(v_{1}, \nu_{2}, \nu_{3}\right) .
\end{aligned}
$$

We show in Fig. 7 the two-scale second-order moments $\left\langle\kappa_{\mathrm{s}}\left(\theta_{\mathrm{s}}\right) \kappa_{\mathrm{s}}\left(\alpha \theta_{\mathrm{s}}\right)\right\rangle$ and $\left\langle M_{\mathrm{ap}}\left(\theta_{\mathrm{s}}\right) M_{\mathrm{ap}}\left(\alpha \theta_{\mathrm{s}}\right)\right\rangle$ for a scale-ratio $\alpha=2,5$, and 10 , at $z_{\mathrm{s}}=1$. A higher $\alpha$ yields a smaller moment because it corresponds to a larger second angular radius $\alpha \theta_{\mathrm{s}}$. We obtain the same level of agreement as for the single-scale variances shown in Fig. 1. In particular, we obtain a good match on small angular scales where the "halo-fit" formula somewhat underestimates the weak-lensing power. On large scales our analytical results are somewhat larger than the data obtained from the numerical simulations. This is due to the missing of large-scale modes in 
P. Valageas et al.: Modeling of weak-lensing statistics. II.
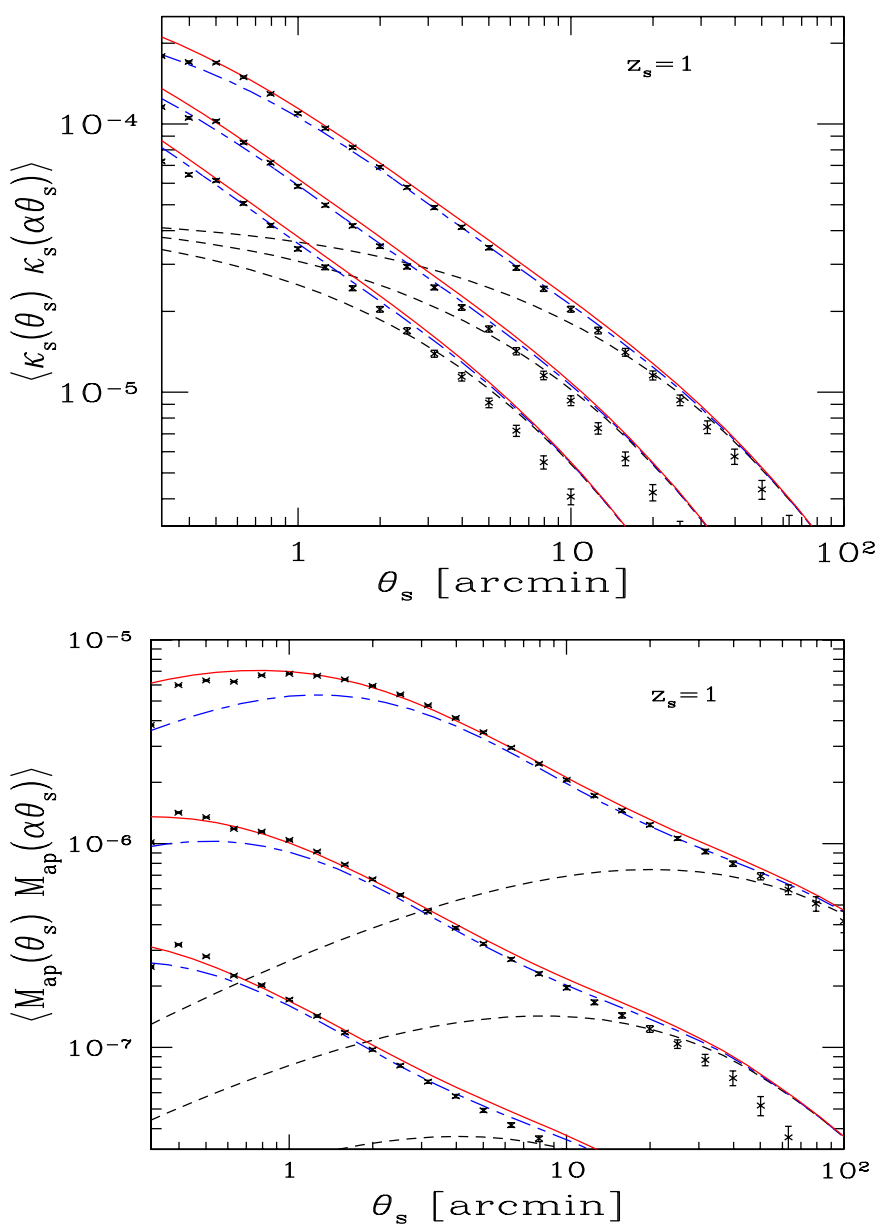

Fig. 7. Two-scale second-order moments $\left\langle\kappa_{\mathrm{s}}\left(\theta_{\mathrm{s}}\right) \kappa_{\mathrm{s}}\left(\alpha \theta_{\mathrm{s}}\right)\right\rangle$ (upper panel) and $\left\langle M_{\mathrm{ap}}\left(\theta_{\mathrm{s}}\right) M_{\mathrm{ap}}\left(\alpha \theta_{\mathrm{s}}\right)\right\rangle$ (lower panel) as a function of $\theta_{\mathrm{s}}$, at $z_{\mathrm{s}}=1$. We show the cases $\alpha=2,5$, and 10 from top to bottom. The symbols are the same as in Fig. 1.

the simulations because of the finite size of the simulation boxes $\left(240 h^{-1}\right.$ and $480 h^{-1} \mathrm{Mpc}$ ) that correspond to 343 and 686 arcmin at $z=1)$. This is more apparent than in the single-scale plots of Fig. 1 because we probe larger scales since the factor $\alpha$ is greater than unity. This again shows the advantage of analytical models such as ours, which are competitive with current ray-tracing numerical simulations to describe a broad range of scales.

We show in Fig. 8 the three-scale third-order moments $\left\langle\kappa_{\mathrm{s}}\left(\theta_{\mathrm{s}}\right) \kappa_{\mathrm{s}}\left(\alpha \theta_{\mathrm{s}}\right) \kappa_{\mathrm{s}}\left(\beta \theta_{\mathrm{s}}\right)\right\rangle$ and $\left\langle M_{\mathrm{ap}}\left(\theta_{\mathrm{s}}\right) M_{\mathrm{ap}}\left(\alpha \theta_{\mathrm{s}}\right) M_{\mathrm{ap}}\left(\beta \theta_{\mathrm{s}}\right)\right\rangle$ for the scale-ratios $\{\alpha, \beta\}=\{2,5\},\{3,9\}$, and $\{5,10\}$, at $z_{\mathrm{s}}=1$. Higher values of $\alpha$ and $\beta$ give a smaller moment since they correspond to larger second and third angular radii $\alpha \theta_{\mathrm{s}}$ and $\beta \theta_{\mathrm{s}}$. We obtain the same level of agreement as for the single-scale third-order moments shown in Fig. 2. In particular, our model recovers the dependence on the ratios $\{\alpha, \beta\}$ and on the scale $\theta_{\mathrm{s}}$ and performs better than the other models studied in this paper. To simplifiy the figures, we show in Fig. 8 only the second-best model, i.e. the " $F_{2, \mathrm{NL}}$ " ansatz (27) using the effective kernel $F_{2, \mathrm{NL}}$ from Scoccimarro \& Couchman (2001) and the power from our model (as in the upper blue dashed line in Fig. 2). Other models show similar behaviors to those found in Fig. 2 for single-scale moments (i.e., a lack of power on moderate and small angular scales). As for the second-order statistics, the underestimate of the weak-lensing signal by the simulations appears at a smaller angle $\theta_{\mathrm{s}}$ than in the single-scale case shown in Fig. 2 because the
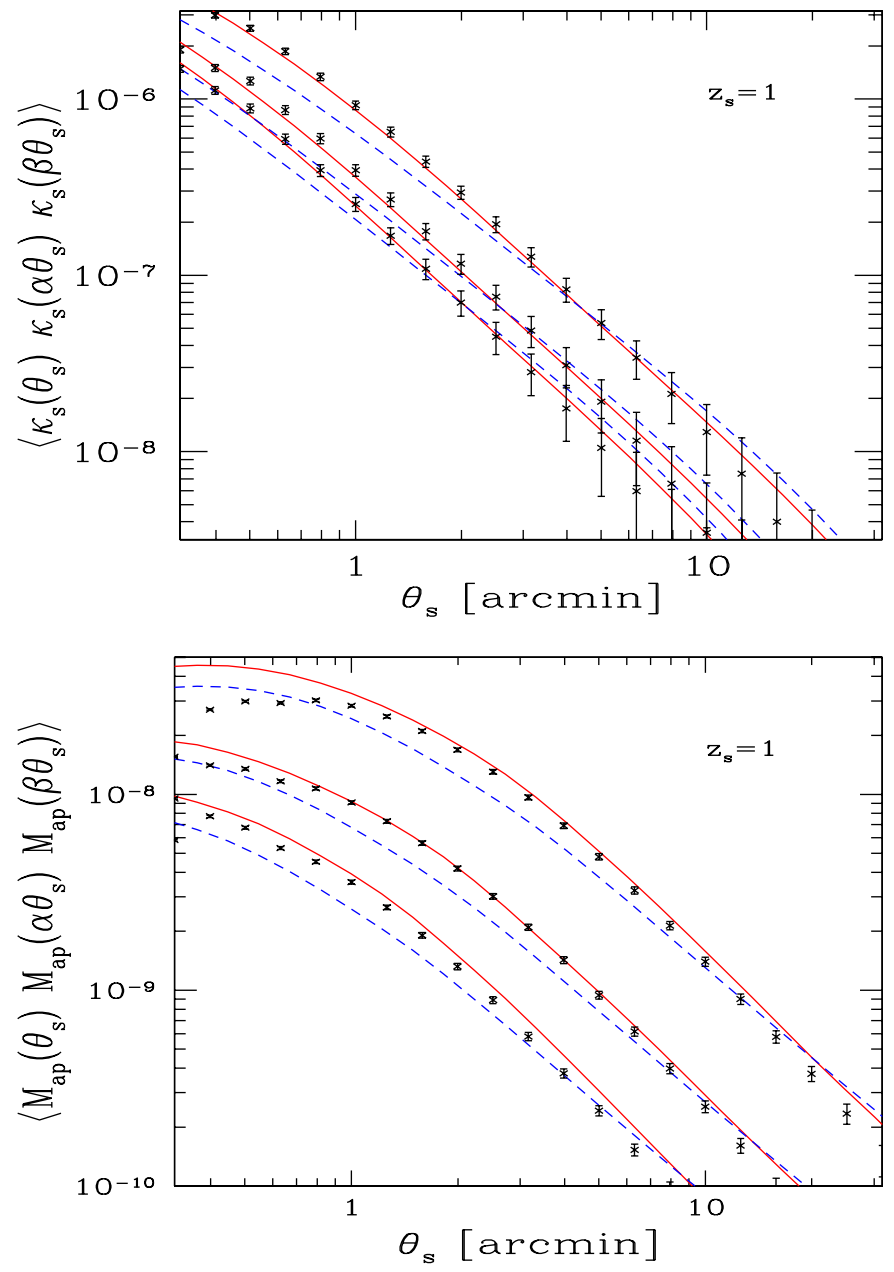

Fig. 8. Three-scale third-order moments at $z_{\mathrm{s}}=1$ as a function of $\theta_{\mathrm{s}}$. Upper panel: $\left\langle\kappa_{\mathrm{s}}\left(\theta_{\mathrm{s}}\right) \kappa_{\mathrm{s}}\left(\alpha \theta_{\mathrm{s}}\right) \kappa_{\mathrm{s}}\left(\beta \theta_{\mathrm{s}}\right)\right\rangle$. Lower panel: $\left\langle M_{\mathrm{ap}}\left(\theta_{\mathrm{s}}\right) M_{\mathrm{ap}}\left(\alpha \theta_{\mathrm{s}}\right) M_{\mathrm{ap}}\left(\beta \theta_{\mathrm{s}}\right)\right\rangle$. We show the cases $\{\alpha, \beta\}=\{2,5\}$, $\{3,9\}$, and $\{5,10\}$ from top to bottom. The blue dashed line is the " $F_{2, \mathrm{NL}}$ " ansatz (27), using the effective kernel $F_{2, \mathrm{NL}}$ from Scoccimarro \& Couchman (2001) and the power from our model (as in the upper blue dashed line in Fig. 2), while the red solid line is our model.

factors $\alpha$ and $\beta$ are larger than unity and increase the sensitivity to larger scales at fixed $\theta_{\mathrm{s}}$.

\section{Conclusion}

We have investigated the performance of current theoretical modeling of the 3D matter density distribution with respect to weak-lensing statistics, focusing on configuration-space statistics, specifically the convergence two-point and three-point correlation functions and the second- and third-order moments of the smoothed convergence and of the aperture mass. As in Paper I, where we studied Fourier-space statistics, we found that a model introduced in previous works (Valageas \& Nishimichi 2011a,b), which combines the (resummed) one-loop perturbation theory with a halo model, fares better than some other recipes based on fitting formulae to numerical simulations or more phenomenological approaches. It yields a reasonable agreement with numerical simulations and provides a competitive approach, because it remains difficult and time-consuming to describe a range of scales that spans three orders of magnitude or more by ray-tracing simulations. 
One advantage of our approach compared with numerical simulations or fitting formulas is that it allows us to decompose the integrated weak-lensing signal over several contributions that are associated with specific properties of the underlying 3D density field. Thus, we can distinguish perturbative terms, which can be derived from perturbation theory, from nonperturbative terms that are associated for instance with one-halo contributions, which depend on the density profile and mass function of virialized halos. This is useful because i) these different terms suffer from different theoretical uncertainties and ii) it allows one to understand which aspects of the matter distribution are probed by weak-lensing statistics, while angular scales vary.

Like for the Fourier-space statistics studied in Paper I and the 3D statistics studied in Valageas \& Nishimichi (2011a,b), we found that including one-loop terms in the perturbative contribution brings a more significant improvement compared with the lowest-order perturbation theory for three-point statistics than for two-point statistics. Then, while large scales are described by these perturbative contributions and small scales by one-halo contributions, the nonperturbative two-halo term that gives an additional contribution to three-point statistics is always subdominant. This is a nice property because this mixed term is more difficult to model and may be less accurate than other contributions (see also Paper I).

Consequently, our model provides reliable predictions for weak-lensing statistics, from small to large scales, and for a variety of cosmologies. It could still be improved in various manners. First, the accuracy of the perturbative contribution may be increased by including higher orders beyond one-loop or by using alternative resummation schemes. Second, the underlying halo model could be refined to include substructures (Sheth 2003; Giocoli et al. 2010), deviations from spherical profiles (Jing \& Suto 2002; Smith et al. 2006), or the effect of baryons (Guillet et al. 2010). Next, the model could be generalized to non-Gaussian initial conditions, which yield distinctive signatures in the bispectrum (Sefusatti et al. 2010).

Acknowledgements. We would like to thank Takashi Hamana for helpful discussions. M.S. and T.N. are supported by a Grant-in-Aid for the Japan Society for Promotion of Science (JSPS) fellows. This work is supported in part by the French "Programme National de Cosmologie et Galaxies" and the FrenchJapanese "Programme Hubert Curien/Sakura, projet 25727TL", the JSPS Coreto-Core Program "International Research Network for Dark Energy", a Grant-inAid for Scientific Research on Priority Areas No. 467 "Probing the Dark Energy through an Extremely Wide and Deep Survey with Subaru Telescope", a Grantin-Aid for Nagoya University Global COE Program, "Quest for Fundamental Principles in the Universe: from Particles to the Solar System and the Cosmos", and World Premier International Research Center Initiative (WPI Initiative), MEXT, Japan. We acknowledge Kobayashi-Maskawa Institute for the Origin of Particles and the Universe, Nagoya University for providing computing resources. Numerical calculations for the present work have been in part carried out under the "Interdisciplinary Computational Science Program" in Center for Computational Sciences, University of Tsukuba, and also on Cray XT4 at Center for Computational Astrophysics, CfCA, of National Astronomical Observatory of Japan.

\section{References}

Albrecht, A., Bernstein, G., Cahn, R., et al. 2006, unpublished [arXiv: astro-ph/0609591]

Bacon, D. J., Refregier, A. R., \& Ellis, R. S. 2000, MNRAS, 318, 625

Bartelmann, M., \& Schneider, P. 2001, Phys. Rep., 340, 291

Benabed, K., \& Scoccimarro, R. 2006, A\&A, 456, 421

Bernardeau, F., Colombi, S., Gaztañaga, E., \& Scoccimarro, R. 2002, Phys. Rep., 367,1

Fu, L., Semboloni, E., Hoekstra, H., et al. 2008, A\&A, 479, 9

Giocoli, C., Bartelmann, M., Sheth, R. K., \& Cacciato, M. 2010, MNRAS, 408, 300

Guillet, T., Teyssier, R., \& Colombi, S. 2010, MNRAS, 405, 525

Hamana, T., Yoshida, N., Suto, Y., \& Evrard, A. E. 2001, ApJ, 561, L143

Hamana, T., Miyazaki, S., Shimasaku, K., et al. 2003, ApJ, 597, 98

Hilbert, S., Hartlap, J., White, S. D. M., \& Schneider, P. 2009, A\&A, 499, 31

Ichiki, K., Takada, M., \& Takahashi, T. 2009, Phys. Rev. D, 79, 023520

Jain, B., Seljak, U., \& White, S. 2000, ApJ, 530, 547

Jarvis, M., Jain, B., Bernstein, G., \& Dolney, D. 2006, ApJ, 644, 71

Jing, Y. P., \& Suto, Y. 2002, ApJ, 574, 538

Kainulainen, K., \& Marra, V. 2011a, Phys. Rev. D, 83, 023009

Kainulainen, K., \& Marra, V. 2011b, Phys. Rev. D, 84, 063004

Kaiser, N. 1992, ApJ, 388, 272

Komatsu, E., Smith, K. M., Dunkley, J., et al. 2011, ApJS, 192, 18

Limber, D. N. 1953, ApJ, 117, 134

Miyazaki, S., Komiyama, Y., Nakaya, H., et al. 2006, Proc. SPIE, 6269, 9

Munshi, D., Valageas, P., van Waerbeke, L., \& Heavens, A. 2008, Phys. Rep., 462, 67

Pan, J., Coles, P., \& Szapudi, I. 2007, MNRAS, 382, 1460

Refregier, A., Amara, A., Kitching, T. D., et al. 2011, A\&A, 528, A33

Sato, M., Hamana, T., Takahashi, R., et al. 2009, ApJ, 701, 945

Sato, M., Ichiki, K., \& Takeuchi, T. T. 2010, Phys. Rev. Lett., 105, 251301

Sato, M., Ichiki, K., \& Takeuchi, T. T. 2011a, Phys. Rev. D, 83, 023501

Sato, M., Takada, M., Hamana, T., \& Matsubara, T. 2011b, ApJ, 734, 76

Schneider, P. 1996, MNRAS, 283, 837

Schneider, P., \& Lombardi, M. 2003, A\&A, 397, 809

Schneider, P., Kilbinger, M., \& Lombardi, M. 2005, A\&A, 431, 9

Schrabback, T., Hartlap, J., Joachimi, B., et al. 2010, A\&A, 516, A63

Scoccimarro, R., \& Couchman, H. M. P. 2001, MNRAS, 325, 1312

Sefusatti, E., Crocce, M., \& Desjacques, V. 2010, MNRAS, 406, 1014

Semboloni, E., Mellier, Y., van Waerbeke, L., et al. 2006, A\&A, 452, 51

Semboloni, E., Schrabback, T., van Waerbeke, L., et al. 2011, MNRAS, 410, 143

Sheth, R. K. 2003, MNRAS, 345, 1200

Shi, X., Schneider, P., \& Joachimi, B. 2011, A\&A, 533, A48

Smith, R. E., Peacock, J. A., Jenkins, A., et al. 2003, MNRAS, 341, 1311

Smith, R. E., Watts, P. I. R., \& Sheth, R. K. 2006, MNRAS, 365, 214

Spergel, D. N., Bean, R., Doré, O., et al. 2007, ApJS, 170, 377

Springel, V. 2005, MNRAS, 364, 1105

Takada, M., \& Jain, B. 2002, MNRAS, 337, 875

Takada, M., \& Jain, B. 2003, MNRAS, 344, 857

Takada, M., \& Jain, B. 2009, MNRAS, 395, 2065

Takahashi, R., Oguri, M., Sato, M., \& Hamana, T. 2011, ApJ, 742, 15

Valageas, P., \& Nishimichi, T. 2011a, A\&A, 527, A87

Valageas, P., \& Nishimichi, T. 2011b, A\&A, 532, A4

Valageas, P., Clerc, N., Pacaud, F., \& Pierre, M. 2011, A\&A, 536, A95

Valageas, P., Sato, M., \& Nishimichi, T. 2012, A\&A, 541, A161

Van Waerbeke, L., Mellier, Y., Erben, T., et al. 2000, A\&A, 358, 30

Van Waerbeke, L., Hamana, T., Scoccimarro, R., Colombi, S., \& Bernardeau, F. 2001, MNRAS, 322, 918

White, M., \& Vale, C. 2004, Astropart. Phys., 22, 19

Wittman, D. M., Tyson, J. A., Kirkman, D., Dell'Antonio, I., \& Bernstein, G. 2000, Nature, 405, 143 